\title{
Gait Monitoring and Walk Distance Estimation With an Accelerometer During 6-Minute Walk Test
}

\author{
Yann Retory, Pascal David, Pauline Niedzialkowski, Carole de Picciotto, Marcel Bonay, and \\ Michel Petitjean
}

\begin{abstract}
BACKGROUND: The 6-min walk test (6MWT) encompasses potential and untapped information related to exercise capacity. However, this test does not yield any information about gait pattern. Recently, we used a ventilatory polygraph to reveal respiratory adaptation during the 6MWT with subjects having high or low body mass index (BMI). In this study, we aimed to determine gait parameters with the same device, which integrates an accelerometer. METHODS: Using a 30-m corridor, steps and U-turns were detected with a custom-made algorithm, compared to video recordings as a reference method, and analyzed offline. From the vertical acceleration signal, we were able to determine cadence and step length, and we could calculate the total distance covered in 6 min (6MWD). We then compared these variables between subjects with low BMI ( $n=13$ subjects) or high BMI ( $n=29$ subjects). RESULTS: Steps and U-turn detection correlated with video results $(r=0.99, P<.001$ for both). The 6MWD calculation was also in line with classical measurements $(r=0.99, P<.001)$. High BMI subjects had a significantly lower 6 MWD, cadence, and step length than controls $(P<.001$ for each). Walking speed was more closely correlated with step length $(r=0.92)$ than with cadence $(r=0.64)$ for both groups. CONCLUSION: Our results demonstrated that a ventilatory polygraph with an embedded accelerometer can be used to detect steps and U-turns, and to calculate 6MWD. This method is sufficiently sensitive to characterize significant BMI-dependent differences in gait pattern during a 6MWT and appears to be a promising tool for routine clinical use. Key words: six-minute walk test; gait analysis; accelerometry; exercise testing; obesity. [Respir Care 2019;64(8):923-930. (c) 2019 Daedalus Enterprises]
\end{abstract}

\section{Introduction}

The quantification of functional capacity in patients presenting with chronic respiratory diseases is an important

\footnotetext{
Dr Retory is affiliated with Centre EXPLOR!, Air Liquide European Homecare Operations Services, Gentilly, France. Drs Retory and Bonay are affiliated with U1179 Inserm, Laboratoire de Physiologie TITAN, Montigny-le-Bretonneux, France. Dr David is affiliated with Service de Médecine Physique et Rééducation, Centre Hospitalier d'Abbeville, Abbeville, France. Drs Retory, de Picciotto, and Bonay, and Ms Niedzialkowski are Service de Physiologie-Explorations Fonctionnelles, Hôpital Ambroise Paré, Assistance Publique-Hôpitaux de Paris, Groupe Hospitalier Paris Ile-de-France Ouest, Boulogne-Billancourt, France. Dr Petitjean is affiliated with Service d'Explorations Fonctionnelles Multidisciplinaires bi-site, Hôpital Antoine Béclère, Groupe Hospitalier ParisSud, Clamart, France; CIAMS, Univ. Paris-Sud, Université Paris-Saclay, 91405 Orsay Cedex, France; and CIAMS, Université d'Orléans, 45067, Orléans, France.
}

The authors have disclosed no conflicts of interest. issue for pulmonologists. These diseases may lead to gasexchange alterations that limit muscle oxygen consumption, especially in muscles involved in locomotion such as the quadriceps. ${ }^{1}$ An alteration of quadriceps endurance has been linked to gait pattern. ${ }^{2}$

The 6-min walk test (6MWT) is a simple, low-cost, and safe exercise test that is used to assess functional exercise capacity by measuring the walking distance covered in 6 min (6MWD). A reduction of total distance, a decrease in hemoglobin saturation as evaluated with a portable pulse

\footnotetext{
Drs Bonay and Petitjean contributed equally to this work.

Correspondence: Michel Petitjean MD PhD, Service d'Explorations Fonctionnelles Multidisciplinaires bi-site, Hôpital Antoine Béclère AP-HP, 157, Rue de la Porte de Trivaux, 92140 Clamart, France. E-mail: michel.petitjean134@gmail.com.
}

DOI: $10.4187 /$ respcare.06144 


\section{AcCelerometer Use During 6MWT}

portable device, and heart rate recovery are the most common indexes of exercise capacity alteration related to respiratory limitations for the 6MWT. ${ }^{3,4}$

Despite its widespread adoption in everyday clinical practice, this functional test does not provide a biomechanical analysis of the gait pattern, which is known to be modified in patients with chronic respiratory diseases. ${ }^{5}$ Instead, mechanical gait parameters are usually explored using biomechanical instrumentation such as a force plate fitted into a walkway or opto-electronic movement-measurement systems. ${ }^{5,6}$ All of these sophisticated techniques require expensive devices, technical expertise, and powerful analysis software, which limit their use in the context of clinical screening with field testing or in a real-life environment. An alternative approach relies on the use of a portable accelerometer. Indeed, an accelerometry-based portable device can objectively provide valid and reliable information about locomotion movement patterns. ${ }^{7}$ Thus, such tools have been used for gait analysis and as a clinical tool in diagnosis and follow-up of gait disorders. ${ }^{8-10}$

In a previous study, we showed the benefits of adding information related to the $6 \mathrm{MWT}$ by using respiratory inductance plethysmography, with an adapted method to describe the breathing pattern in lean and overweight healthy adults during the 6MWT. ${ }^{11}$ Hence, we hypothesized that an integrated biomechanical analysis of gait combined with ventilatory monitoring during the $6 \mathrm{MWT}$ in a population of overweight subjects would yield relevant biomechanical information. A description of gait pattern alteration would help describe the impact of ventilatory limitation.

Respiratory recording devices, such as the portable monitor that we described previously, can be embedded in an upper trunk-mounted triaxial accelerometer and may prove to be a good candidate for gait monitoring during the 6MWT. ${ }^{11,12}$ Accordingly, the primary aim of this study was to establish the possibility of obtaining relevant basic gait parameters during the specific conditions of the 6MWT with a portable monitor placed at a thoracic position. As previously reported for breathing pattern, ${ }^{11}$ the second aim was to explore this method's sensitivity when comparing gait parameters between subjects with low or high body mass index (BMI).

\section{Methods}

\section{Participants}

A total of 52 subjects (41 women, 11 men) volunteered to participate in this study; 10 subjects were included in a video-validation group, while the 42 remaining subjects were divided into 2 subgroups according to BMI. To determine BMI, body weight was measured to the nearest $0.1 \mathrm{~kg}$ using a scale (Seca Model 750 VIVA, Hamburg, Germany), and height was measured to the nearest $0.1 \mathrm{~cm}$ with a wall-mounted stadiometer (NM Medical, Asnières,

\section{QUICK LOOK}

\section{Current knowledge}

The 6-min walk test (6MWT) in its classical design gives useful information on functional capacity. However, gait parameters are not evaluated by this test.

\section{What this paper contributes to our knowledge}

This study aimed at describing gait parameters and evaluating walk distance during 6MWT with an accelerometer in thoracic position. This method allows calculations of step length, cadence and distance walked by processing step events and U-turns. Comparison between high and low BMI subjects reveals a possible discrimination based on gait parameters. Those results are promising for the evaluation of gait in routine with 6MWT.

France). Thirteen subjects with BMI $<25 \mathrm{~kg} / \mathrm{m}^{2}$ and 29 subjects with BMI $>30 \mathrm{~kg} / \mathrm{m}^{2}$ were included in the low and the high BMI groups, respectively (Table 1).

Exclusion criteria included a clinical history of metabolic or respiratory disease, inability to walk, and smoking. This study was approved by the Institutional Review Board of the French Learned Society for Respiratory Medicine (Société de Pneumologie de Langue Française: $\mathrm{n}^{\circ}$ 2015-23) and was conducted in accordance with the last release of the Declaration of Helsinki. All subjects gave their written consent to participate.

\section{Recordings}

As in our previous studies, a Nox-T3 thoracic polygraph system (Nox Medical, Reykjavík, Iceland) was used to record all signals (Fig. 1). The Nox-T3 device also has an

Table 1. Demographic Characteristics of Study Participants

\begin{tabular}{|c|c|c|c|c|}
\hline & Control & Non-overweight & Overweight & $P$ \\
\hline Age, $y$ & $43.8(12.8)$ & $42.2(13.6)$ & $38.4(10.7)$ & .39 \\
\hline Weight, kg & $67.3(12.1)^{*}$ & $63.4(8.6)^{*}$ & $121(18.7)$ & $<.001$ \\
\hline Height, m & $166.7(6.8)$ & $167.2(7.5)$ & $165.9(8.8)$ & .88 \\
\hline $\begin{array}{l}\text { Body mass } \\
\text { index, } \mathrm{kg} / \mathrm{m}^{2}\end{array}$ & $24(2.5)^{*}$ & $22.6(1.8)^{*}$ & $43.8(5.4)$ & $<.001$ \\
\hline $\begin{array}{l}\text { Male/female } \\
\quad \text { ratio }\end{array}$ & $3 / 7$ & $4 / 9$ & $4 / 25$ & .34 \\
\hline \multicolumn{5}{|c|}{$\begin{array}{l}\text { Control } n=10 \text {; Non-overweight } n=13 \text {; Overweight } n=29 \text {. Values are mean (SD). One-way } \\
\text { analyses of variance were used to compare group means for the different demographic } \\
\text { variables. Post-hoc analysis consisted of paired } t \text { tests with Bonferroni correction. } * P<.05 \\
\text { versus overweight. The chi-square test was used to compare the male/female ratios among the } \\
\text { groups. }\end{array}$} \\
\hline
\end{tabular}




\section{AcCelerometer Use During 6MWT}
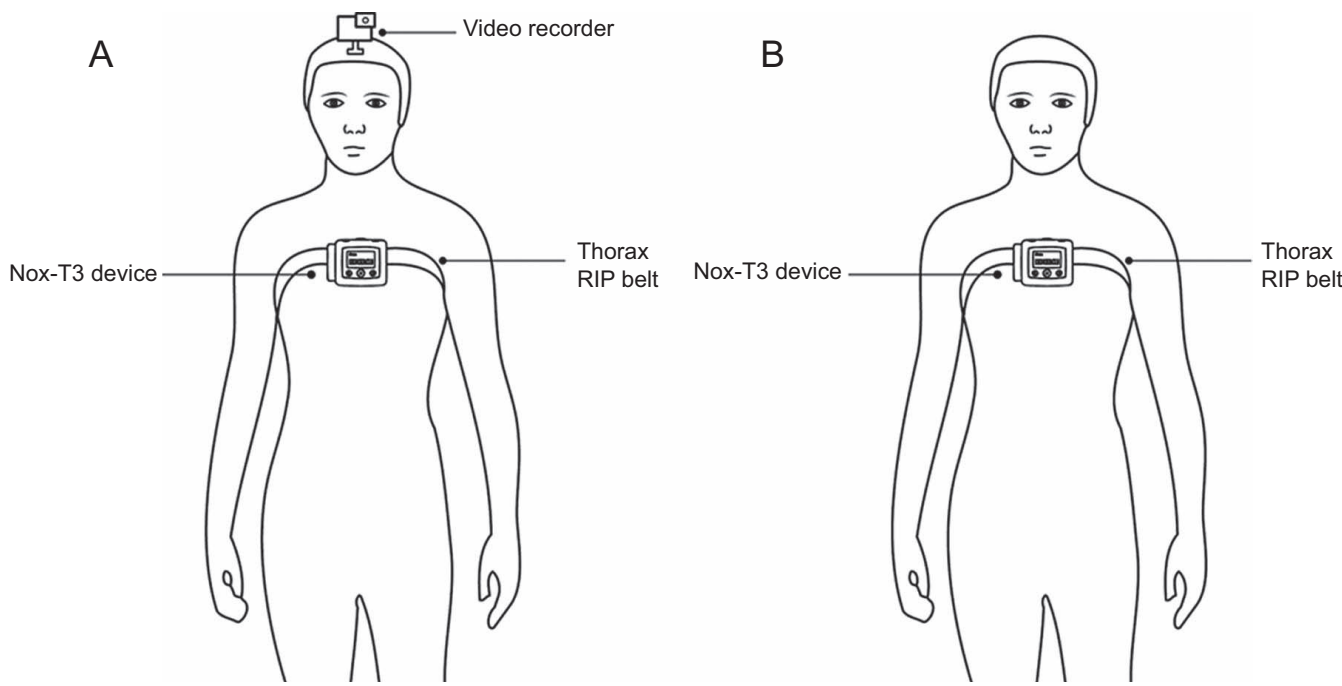

Fig. 1. The Nox-T3 equipment as worn by a participant. Total carrying mass is $88 \mathrm{~g}$. A: Includes video camera. B: Without video camera.

embedded triaxial accelerometer (range: $\pm 2 \mathrm{~g}$ ), which can continuously measure upper trunk accelerations. According to the characteristics of the Nox-T3 device, accelerometric signals were sampled at $10 \mathrm{~Hz}$. The raw data were transmitted onto a computer via the Noxturnal software (version 3.2) for offline processing.

A portable video recorder (Geonaute G-eye discover, Groupe Decathlon, Mons, France), wearable on the forehead was used as a reference to record steps (Fig. 1A). The video recorder had a resolution of 720 pixels with a frame rate of $30 \mathrm{frames} / \mathrm{s}$. Velocity was calculated as the distance covered between consecutive U-turns divided by the corresponding duration.

\section{Experimental Procedures}

Gait was assessed during the 6MWT, which was performed according to guidelines from the American Thoracic Society. ${ }^{13}$ Only subjects from the first group were equipped with the video camera on their forehead (Fig. 1A) for validation sessions with video.

\section{Data Processing}

As described in our previous papers, ${ }^{11,12}$ data files were extracted and converted into ASCII files for offline analysis. Signal processing was performed with custom routines written in Matlab (Release 2015b, The Mathworks, Inc., Natick, Massachusetts). Among the 3 directions of acceleration, vertical acceleration $(Z)$ provided a better graphical recognition of steps in all subjects. Thus, a raw $\mathrm{Z}$ signal was bandpassfiltered between $0.9 \mathrm{~Hz}$ to remove the direct current (DC) component, which was mainly the influence of gravity, and $2.5 \mathrm{~Hz}$ to remove tissue vibration contamination by using a 5-order Butterworth filter (Fig. 2A). Considering the cyclical nature of the collected accelerometric signal and gait, we assigned step-event detection to all peaks found in the filtered signal. As such, a peak detection function was used to count the steps, defined by the local maximum of the filtered acceleration signal (Fig. 2B). U-turns were identified as steps characterized by a significant deceleration in comparison with other steps. ${ }^{14}$ A moving root mean square envelope of a Z-filtered signal with a sliding window of 3 consecutive points was used for detection. This envelope was characterized by 2 curves: an upper curve (Z-upper) and a lower curve (Zlower) (Fig. 2C). A threshold value was set at the 95th percentile of the Z-filtered values during the 6MWT, below which the values were omitted (Fig. 2D). A refractory period of $10 \mathrm{~s}$ was respected, during which 2 consecutive U-turns could not be labeled. Average step lengths were then calculated by dividing the distance between 2 consecutively detected U-turns, namely at $30 \mathrm{~m}$, by the median of the number of steps needed to cover it. The 6MWD was calculated as the product of the global number of steps detected during the 6MWT by the median step length, and this was compared to the distance actually measured.

\section{Video Validation}

The individual video recordings of the 6MWT were viewed by 2 observers blinded to each other. They were instructed to count steps during a predetermined period of time $(10 \mathrm{~s}, 20 \mathrm{~s}, 40 \mathrm{~s}, 50 \mathrm{~s}, 80 \mathrm{~s}$, and $160 \mathrm{~s})$ without overlapping from the onset to the end of the 6MWT. They were also instructed to time U-turn occurrences. The data obtained from the 2 observers were compared to those of the automatized detection process for steps and U-turns. 

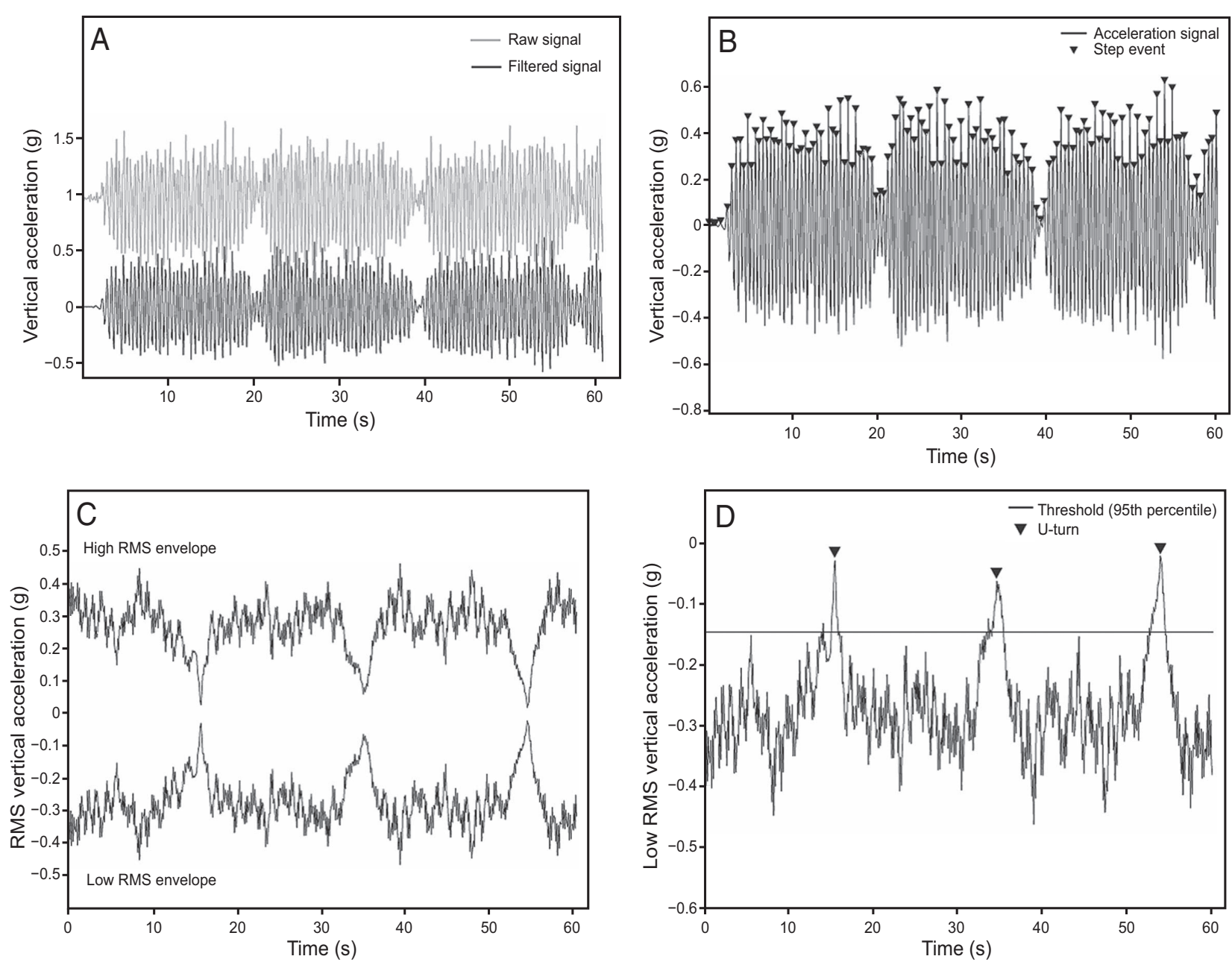

Fig. 2. Vertical accelerometric signal processing protocol. See text for further details.

\section{Statistical Analysis}

Bland-Altman analysis, including calculation of mean bias between each testing protocol (automatized detection process from polygraph, video via observer 1 , and video via observer 2) and standard deviation (SD) of bias (bias $\pm 2 \mathrm{SD}$ ), was used to evaluate the performance of the proposed method. ${ }^{15}$ Data from the polygraph were checked for normality and for equality of variance. A multivariate analysis of variance (MANOVA) with repeated measures was used to analyze changes in the outcome measures as a function of group status and time. For effects that were statistically significant in the MANOVA, follow-up analyses included a univariate analysis of variance. Using the data from all subjects, Bravais-Pearson correlation coefficients (r) were calculated to evaluate potentially linked parameters. A linear regression was established to characterize the relationship between the 2 methods for determining 6MWD. Differences were considered significant when $P<.05$. Data are presented as mean $\pm 1 \mathrm{SD}$.
Statistics corresponding to validation results were assessed with GraphPad Prism version 5.01 (San Diego California), whereas those corresponding to determination of gait parameters of subjects with high or low BMI were calculated with Sigmastat (SigmaPlot, Systat Software, San Jose, California).

\section{Results}

\section{Technical Validation}

As shown in Figure 3A, there was a significant correlation between the number of steps detected with the accelerometer analysis and the video observation from both observers ( $r=0.99, P<.01$ for both observers). However, Bland and Altman analysis (Fig. 3B, 3C and 3D) revealed biases between the two observers and between each observer and the polygraph, which are in the same range $(-0.7 \pm 3.5$ steps; $1.2 \pm 2.9$ steps and $0.5 \pm 2.5$ steps, respectively). 

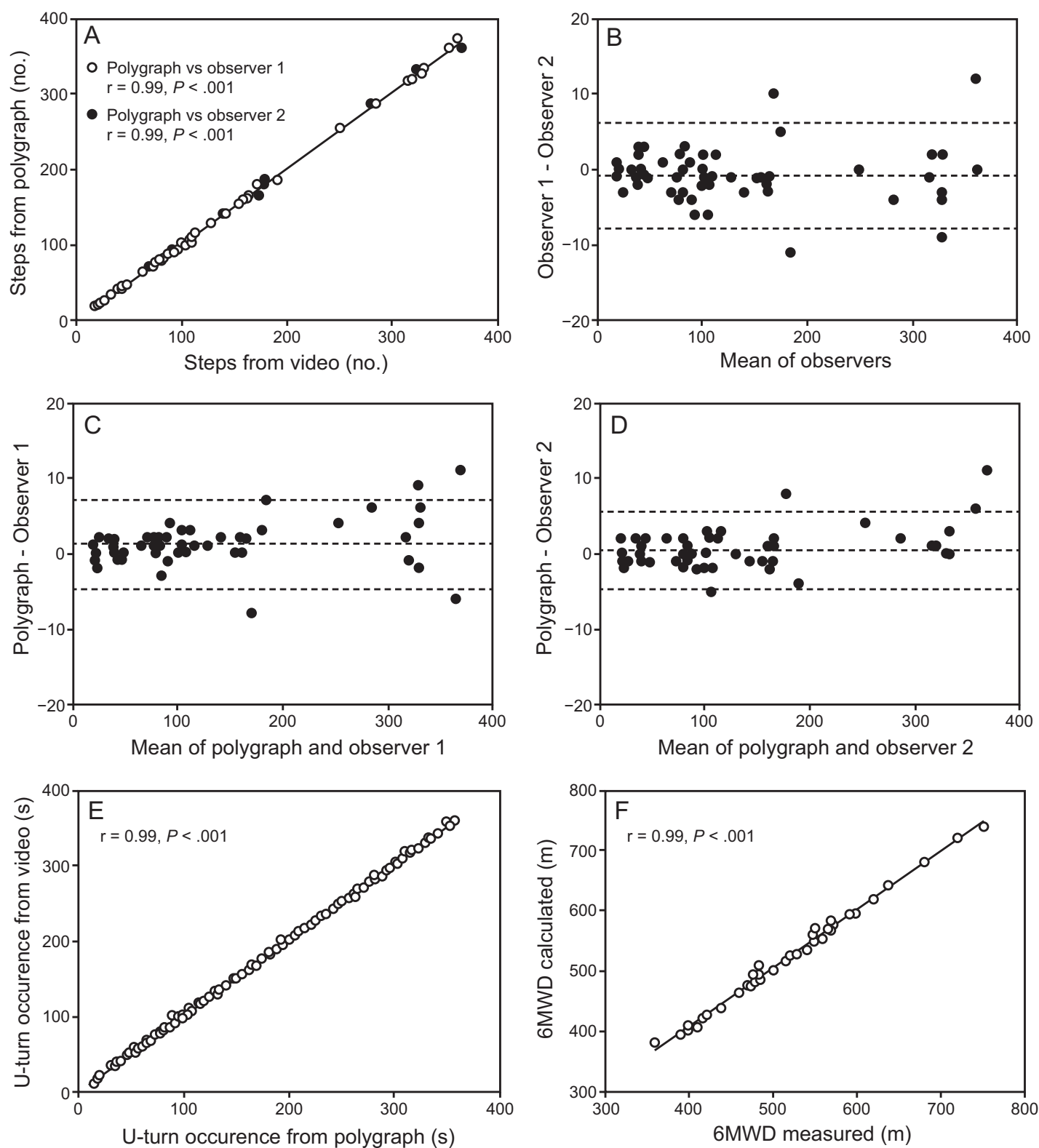

Fig. 3. A: Relationship between the number of steps detected by the polygraph and video. B, C, D: Bland-Altman difference plots for the number of step obtained from the polygraph and the video (observer 1 and observer 2). The mean bias, representing the mean difference between 2 testing protocols, appears on the graph as a horizontal dashed line, with the upper and lower lines representing the limits of agreement (bias $\pm 2 \mathrm{SD}$ ). E: Correlation between U-turn occurrences detected in the polygraph and video. F: Correlation between the 6-min walk distance assessed in the polygraph and video. Linear regression fit (black line), $r$, and $P$ values are reported.

There was a significant correlation between the occurrences of U-turns detected by the first observer and the second observer $(r=0.99, P<.01)$. As a result, correlations were identical and significant $(r=0.99, P<.01)$ between U-turns detected with the accelerometer and those detected by the 2 observers (Fig. 3E), with mean biases of $<1 \mathrm{~s}(-0.68 \pm 2.37 \mathrm{~s}$ and $-0.64 \pm 2.33 \mathrm{~s})$ as determined with revealed Bland-Altman analyses. The sensitivity (ie, the number of steps correctly considered to be implied among the 181 U-turns tested), was $95.7 \%$, whereas the specificity (ie, number of steps correctly considered not implied in U-turns among the 7,231 steps tested) was $99.9 \%$.

There was a significant positive correlation $(r=0.99$, $P<.01)$ characterizing the relationship between 6MWD determined classically and with those of our data treatment (Fig. 3F). For this relationship, the slope was close to the identity line (0.97) with an intercept of $19.2 \mathrm{~m}$ and an average bias of $3.9 \pm 7.7 \mathrm{~m}$. 


\section{Subjects with Low or High BMI}

There was no significant difference in biometric characteristics between groups except for weight and BMI (Table 1). Nevertheless, the 6MWD for subjects with high BMI was significantly lower than the 6MWD for subjects with low BMI $(P<.001)$. As shown in Figure 4A, considering velocity during the $6 \mathrm{MWT}$, there was a BMI effect (explaining $38.5 \%$ of the total variation, $P<.01$ ) without a time effect. Tukey pairwise comparisons demonstrated significantly lower values for the high-BMI group at each minute of the test in comparison with the low-BMI group $(P<.01)$. For cadence (Fig. 4B), there was also a BMI effect (explaining 22.3\% of the total variation, $P<.01$ ), with lower values for the high-BMI group without a time effect. Furthermore, there was a BMI effect at each minute of the test $(P<.01, P<.01, P<.05$, $P<.01, P<.01, P<.01$, respectively). Step length (Fig. $4 \mathrm{C}$ ) was also influenced by BMI (explaining $27.4 \%$ of the total variation, $P<.01$ ), with high-BMI subjects showing lower values without any significant time effect. In addition, velocity was better correlated with step length $(r=0.92)$ than with cadence $(r=0.64)$.

\section{Discussion}

\section{Technological Aspects}

Our results demonstrated the possibility of monitoring basic gait parameters with a classical ventilatory polygraph placed at a thoracic position during the 6MWT. Despite the fact that this was not our primary goal, we also confirmed that this is a simple tool to calculate with confidence the total distance covered during the 6MWT. As reported in the literature, accelerometry has been primarily used to estimate gait parameters. ${ }^{8}$ In this study, the device was located at the thorax, which is unusual (ie, accelerometers typically are placed near the center of mass in the lumbar position ${ }^{16}$ or on the ankle ${ }^{17}$ ), but this related to the accelerometer on the ventilatory polygraph. Kavanagh et al ${ }^{18}$ described accelerometric signals produced by sensors located in the occipital position and near the L3 spinous process and found a strong correlation between signals produced in both locations. Representations of head accelerations reported by Kavanagh et $\mathrm{al}^{18}$ were close to those recorded in our study during the 6MWT. This indicates that the thoracic location seems suitable when step detection is required. Another concern is the detection of steps by way of the acceleration signal, which only measures in the vertical direction. Our device actually allows recording of mediolateral and anteroposterior components. Kavanagh et $\mathrm{al}^{18}$ found a better correlation between the head and trunk vertical component of acceleration than for the mediolateral and anteroposterior components. Further-
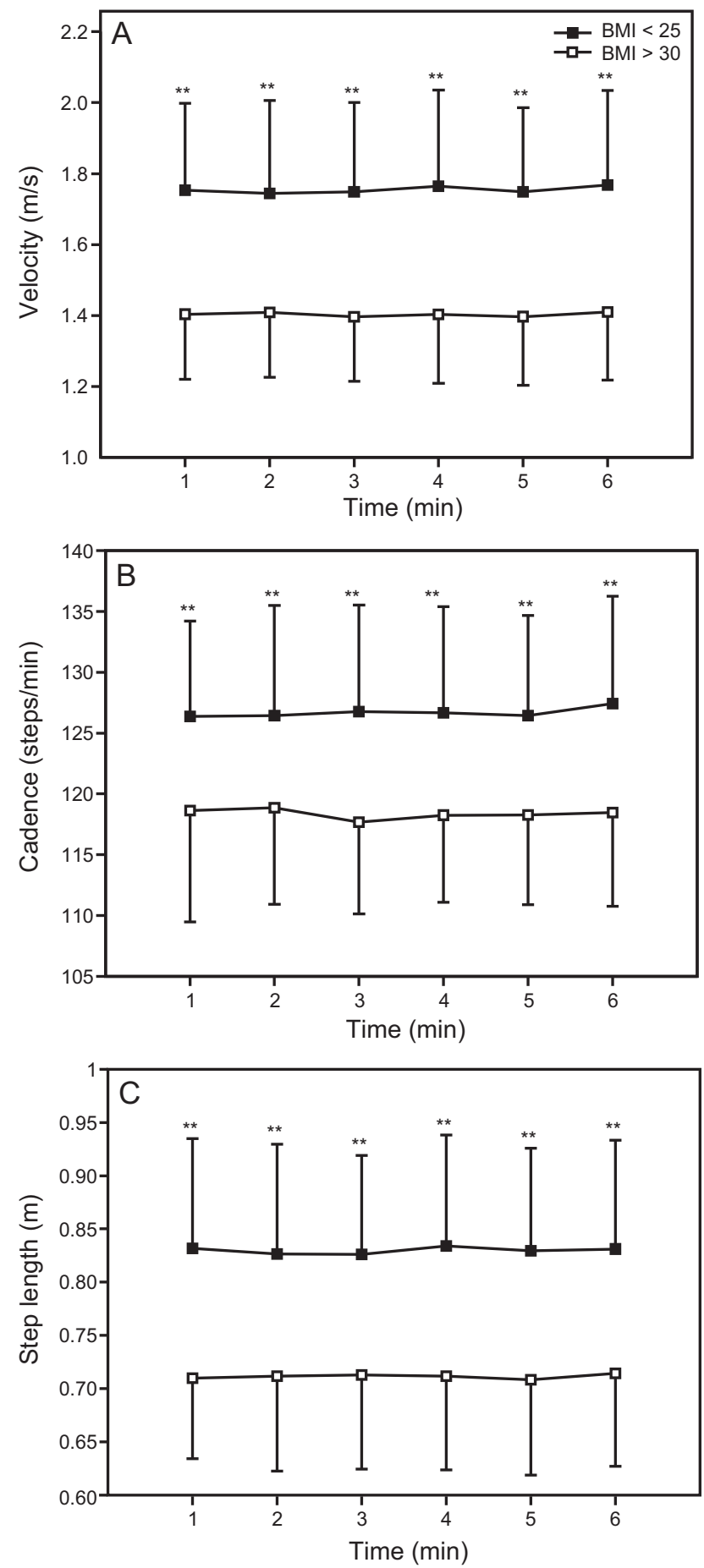

Fig. 4. Spatiotemporal gait parameters (A: velocity; B: cadence; C: step length) for the control group $(n=13)$ and the overweight group $(n=29)$ during the 6-min walk test. Statistical differences were found between groups $\left({ }^{* *} P<.001\right)$. No statistical differences were found within a group over time $(P>.05)$. $P$ values are results of a $2 \times 6$ (Group $A \sim$ Time) repeated measures analysis of variance with post hoc Tukey testing analysis. Values are mean \pm 1 SD. 


\section{AcCelerometer Use During 6MWT}

more, the 2 vertical accelerometer signals exhibited better phase congruence. Kavanagh et al ${ }^{18}$ interpreted these results by suggesting that the trunk does not play a major role as a shock absorber in the vertical direction. Thus, we hypothesized that the vertical component of acceleration remains the most appropriate signal to estimate center-ofmass accelerations during walking when placing the sensor away from the center of mass.

Another point was the sampling frequency of our device, which was lower $(10 \mathrm{~Hz})$ than what is generally used for gait analysis. ${ }^{19}$ Indeed, sampling rates from $25 \mathrm{~Hz}$ up to $200 \mathrm{~Hz}$ are have been reported in other studies. ${ }^{19}$ However, Huang et $\mathrm{al}^{20}$ successfully used a $10 \mathrm{~Hz}$ sampling frequency to count steps with an accuracy of 93-96\%. This seems compatible with the Shannon Theorem, which states that the minimal sampling rate of a signal has to be at least twice the maximum frequency of the target phenomenon. ${ }^{21}$ This indicates that this system cannot accurately describe a gait compound higher than $5 \mathrm{~Hz}$, but it does seem capable of detecting cadence, which is much lower than $5 \mathrm{~Hz}$.

Cutoff frequencies for the bandpass filtering selected in this study are motivated by rejection of respiratory motion artifacts and tissue motion artifacts induced by walking. Indeed, the lower cutoff of $0.9 \mathrm{~Hz}$ was set to avoid respiratory movement contamination, as well as the DC component, namely gravity. The upper cutoff at $2.5 \mathrm{~Hz}$ was determined to limit contamination by vibration of fat tissue in subjects with high BMI. Indeed, we have reported that artifact occurrence was proportional to individual BMI values. ${ }^{11}$ Perhaps $2.5 \mathrm{~Hz}$ appears too low, and, as indicated by Capela et al, ${ }^{19}$ a cutoff frequency below $4 \mathrm{~Hz}$ would miss some steps with an accelerometer placed in a lumbar position. To our knowledge, the particular setup used in our study (ie, cutoff frequencies with this device location) had never been tested before in the context of the 6MWT. It is noteworthy that this filtering limits the ability to accurately determine phases of gait, such as swing or sway periods. However, in our experimental paradigm, the cutoff frequency was not detrimental in estimating the number of steps.

\section{Performance}

In our methods, U-turn detection is important because this is part of the 6MWT protocol, and also because this variable is taken into account when calculating mean step length. Gyroscopic sensors in wearable smartphones have been used in previous studies to detect U-turns. ${ }^{19} \mathrm{We}$ empirically observed that a marked deceleration occurred during each U-turn. This observation is well supported by the close linear relationship between velocity and the curvature of a locomotor trajectory as described by Vieilledent et al, ${ }^{14}$ which supports the observation that a reduction of speed and acceleration occurs during U-turns. Our results, based on a deceleration assessment, were sufficient to confidently detect U-turns with good specificity and sensibility as validated with our video recordings.

Given the specificity of the 6MWT protocol, which includes turns within at least $30 \mathrm{~m}$ of a hallway, we used the distance between turns and the number of steps needed to cover this length to determine mean step length, and we then multiplied this by the global step number to estimate a $6 \mathrm{MWD}$ value. ${ }^{13}$ Step detection is an important concern, and our data demonstrated adequate step detection. It is likely that these results are due to the particular conditions of the 6MWT. Indeed, subjects try to walk as far as possible during $6 \mathrm{~min}$, so they naturally adopted a very regular locomotor pattern at a speed slightly higher than a spontaneous or preferred walking speed.

Having a few errors did not impair the global results. This is likely due to the fact that we used median step length values rather than means in the calculation of 6MWD. This prevented inclusion of possible aberrant values that might occur if adding or omitting one or several U-turns. Both step and U-turn detection showed satisfactory results with very few errors. When calculating 6MWD, these errors may be cumulative, but global accuracy seems to be suitable for clinical distance estimation with an average bias of $3.9 \mathrm{~m} \pm 7.7 \mathrm{~m}$.

Finally, our results showed that automatic 6MWD determination and obtaining basic gait parameters are readily feasible in addition to the ventilatory monitoring during a $6 \mathrm{MWT}$ as proposed in our previous study. ${ }^{11}$

\section{Clinical Considerations}

The standardized format of the 6MWT contributes to worldwide applicability. Due to its simplicity, however, this test does not give information on ventilatory adaptation or on locomotor pattern. In this study, we aimed to obtain gait parameters such as cadence, step length, and velocity with the same device. It is well known that patients with high BMI have lower 6MWD as well as lower cadence and step length. ${ }^{5}$ Knowing that there were no significant differences for height, age, and sex ratio, our results showed that velocity, cadence, and step length were significantly lower in subjects with high BMI than in controls at each minute of the test without any time effect. This suggests that the locomotor adaptation in subjects with high BMI is meaningful and may be linked to ventilation or cardiac factors that result in different adaptation strategies during exercise.

\section{Conclusion}

We conclude that locomotor monitoring of the 6MWT provides a useful tool to better characterize gait parameter 


\section{ACCELEROMETER Use During 6MWT}

impairments related to high BMI. Validation of such information can enrich ventilatory monitoring. Moreover, this method could be used with different patient populations with respiratory diseases such as COPD, pulmonary fibrosis, or pulmonary hypertension. The ability to measure walk-distance reduction along with a correlation in respiratory limitation and gait-parameter alteration may be of practical clinical use.

\section{ACKNOWLEDGMENTS}

We thank Mr Flavien Mousin, Data Manager, for reviewing the statistical procedure, and the anonymous English reviewer from Servicescape who edited the English manuscript prior to its submission.

\section{REFERENCES}

1. Coronell C, Orozco-Levi M, Méndez R, Ramírez-Sarmiento A, Gáldiz $\mathrm{JB}$, Gea J. Relevance of assessing quadriceps endurance in patients with COPD. Eur Respir J 2004;24(1):129-136.

2. Murdock GH, Hubley-Kozey CL. Effect of a high-intensity quadriceps fatigue protocol on knee joint mechanics and muscle activation during gait in young adults. Eur J Appl Physiol 2012;112(2):439449 .

3. Enright PL. The six-minute walk test. Respir Care 2003;48(8):783785.

4. Cahalin LP, Arena R, Labate V, Bandera F, Lavie CJ, Guazzi M. Heart rate recovery after the 6-min walk test rather than distance ambulated is a powerful prognostic indicator in heart failure with reduced and preserved ejection fraction: a comparison with cardiopulmonary exercise testing. Eur J Heart Fail 2013;15(5):519-527.

5. da Silva-Hamu TC, Formiga CK, Gervásio FM, Ribeiro DM, Christofoletti G, de França Barros J. The impact of obesity in the kinematic parameters of gait in young women. Int J Gen Med 2013; 24(6):507-13.

6. Hortobágyi T, Herring C, Pories WJ, Rider P, Devita P. Massive weight loss-induced mechanical plasticity in obese gait. J Appl Physiol 2011;111(5):1391-1399.

7. Plasqui G, Bonomi AG, Westerterp KR. Daily physical activity assessment with accelerometers: new insights and validation studies. Obes Rev 2013;14(6):451-462.
8. Kavanagh JJ, Menz HB. Accelerometry: a technique for quantifying movement patterns during walking. Gait Posture 2008;28(1):1-15.

9. Nordstrøm M, Hansen BH, Paus B, Kolset SO. Accelerometer-determined physical activity and walking capacity in persons with Down syndrome, Williams syndrome and Prader-Willi syndrome. Res Dev Disabil 2013;34(12):4395-4403.

10. Matsushima A, Yoshida K, Genno H, Murata A, Matsuzawa S, Nakamura $\mathrm{K}$, et al. Clinical assessment of standing and gait in ataxic patients using a triaxial accelerometer. Cerebellum Ataxias 2015;6:2-9.

11. Retory Y, de Picciotto C, Niedzialkowski P, Petitjean M, Bonay M. Body mass index-dependent ventilatory parameters from respiratory inductive plethysmography during 6-minute walk rest. Respir Care 2016;61(4):521-528

12. Retory Y, Niedzialkowski P, de Picciotto C, Bonay M, Petitjean M. New respiratory inductive plethysmography (RIP) method for evaluating ventilatory adaptation during mild physical activities. PLoS One 2016;11(3):1-12.

13. ATS Committee on Proficiency Standards for Clinical Pulmonary Function Laboratories: ATS statement: guidelines for the six-minute walk test. Am J Respir Crit Care Med 2002;166(1):111-117.

14. Vieilledent S, Kerlirzin Y, Dalbera S, Berthoz A. Relationship between velocity and curvature of a human locomotor trajectory. Neurosci Lett 2001;305(1):65-69.

15. Bland JM, Altman DG. Statistical methods for assessing agreement between two methods of clinical measurement. Lancet 1986;1(8476): 307-310.

16. Moe-Nilssen R, Helbostad JL. Estimation of gait cycle characteristics by trunk accelerometry. J Biomech 2004;37(1):121-126.

17. Lee JA, Cho SH, Lee YJ, Yang HK, Lee JW. Portable activity monitoring system for temporal parameters of gait cycles. J Med Syst 2010;34(5):959-966.

18. Kavanagh JJ, Barrett RS, Morrison S. Upper body accelerations during walking in healthy young and elderly men. Gait Posture 2004; 20(3):291-298.

19. Capela NA, Lemaire ED, Baddour N. Novel algorithm for a smartphone-based 6-minute walk test application: algorithm, application development, and evaluation. J Neuroeng Rehabil 2015;12(19):1-13.

20. Huang Y, Zheng H, Nugent C, McCullagh P, Black N, Burns W, et al. An orientation-free adaptive step detection algorithm using a smart phone in physical activity monitoring. Health Technol 2012; 2(4):249-258.

21. Shannon CE. Communication in the presence of noise. Proc IRE 1949;37(1):10-21. 